\title{
Guillain-Barré syndrome in times of pandemics
}

\author{
Sonja E Leonhard (1) , ${ }^{1}$ David R Cornblath, ${ }^{2}$ Hubert P Endtz, ${ }^{3,4}$ \\ James J Sejvar, ${ }_{1}^{5}$ Bart C Jacobs ${ }^{6}$
}

\begin{abstract}
The SARS-CoV-2 pandemic is the last in line of several epidemics of infectious diseases that have been linked to the Guillain-Barré syndrome (GBS). As threats of epidemics of emerging infectious diseases persist, this is the time to learn from the past and to advance our response to future outbreaks in terms of research and management of GBS.
\end{abstract}

In the past decade, the world confronted several pandemics of emerging infectious diseases including Zika virus and most recently Severe Acute Respiratory Syndrome Coronavirus 2 (SARS-CoV-2). One of the neurological complications reported in relation to these infectious diseases is the Guillain-Barré syndrome (GBS), a rapidly progressive immunemediated polyradiculoneuropathy that can cause paresis in all limbs, cranial and respiratory muscles. ${ }^{1-3}$ Approximately 20\% require admission at an intensive care unit (ICU), and 2\%-12\% die, depending on the care available. ${ }^{4}$

In the past, research responses investigating a possible link between GBS and outbreaks of infectious diseases or vaccines have been delayed. This is problematic as healthcare institutions need to be able to prepare for increased incidences in patients with GBS, and public health personnel need to identify any possible mitigating factors. History now seems to repeat itself when case reports of SARS-CoV-2-related GBS are mounting, and disquiet over a possible association increases. As threats of epidemics of emerging infectious diseases persist, this is the time to learn from the past and to advance our response to future outbreaks in terms of research and management of GBS.

\footnotetext{
${ }^{1}$ Neurology, Erasmus MC, Rotterdam, The Netherlands ${ }^{2}$ Neurology, Johns Hopkins University, Baltimore, Maryland, USA

${ }^{3}$ Medical Microbiology \& Infectious Diseases, Erasmus MC, Rotterdam, The Netherlands

${ }^{4}$ Fondation Mérieux, Lyon, France

${ }^{5}$ National Center for Emerging and Zoonotic Infectious Diseases, Centers for Disease Control and Prevention, Atlanta, Georgia, USA

${ }^{6}$ Neurology and Immunology, Erasmus MC, Rotterdam, The Netherlands
}

Correspondence to Dr Bart C Jacobs, Neurology and Immunology, Erasmus MC, Rotterdam 3015 GD, The Netherlands; b.jacobs@erasmusmc.nl

\section{CHALLENGES AND PROSPECTS IN RESEARCH PREPAREDNESS}

The first aims when studying a possible link between an infectious agent and GBS are to determine if a true association exists and to determine the impact in terms of frequency and severity. During an outbreak, observational cohorts are set up rapidly by clinicians, some of whom may lack experience in diagnosing and managing GBS due to the need to quickly mobilise personnel. These studies are often done at a single centre and not harmonised with GBS research from other centres, which can result in missing out of important clinical information.

How can one ensure a high-quality study within the limited time frame afforded by an infectious disease epidemic? Many hurdles must be overcome before recruitment can be started, and accurate and sufficient data collection is complex. Here, we list the most important hurdles and provide suggestions on how to deal with them.

\section{Study design: surveillance and case- control studies}

To determine the impact in terms of frequency, a reliable and international surveillance platform for GBS incidence during and between epidemics, either active or passive, should be in place to define the background incidence and to detect an increase in cases. A surveillance system for acute flaccid paralysis (AFP) in children under the age of 15 was set up to eradicate polio and is operative globally (http://polioeradication.org/). The international community may benefit from introducing AFP surveillance for all ages or for GBS specifically.

To determine an association between GBS and an infectious agent, a cohort study with a case-control design is necessary. A predefined research protocol should be developed that is feasible in different healthcare infrastructures and easy to activate and use, to ensure a high-quality study within a limited time frame. Critical requirements for the study include clear case definitions for GBS and the collection of data on the clinical and electrophysiological phenotype, as this can be associated with a specific infectious agent and may provide evidence of an association. To study the impact for patients, outcome of at least 6-12 months with validated outcome measures should be recorded.

Such a protocol would be supported by a network of neurologists, such as the Inflammatory Neuropathy Consortium of the Peripheral Nerve Society, and can be based on the protocol of the International GBS Outcome Study, that is running in 19 countries and is also used by other research groups. ${ }^{56}$ Existing networks such as The Global Health Network could help to make the existence of such a protocol widely known. ${ }^{7}$ Inspiration can be drawn from large international research consortia on infectious diseases, such as the International Severe Acute Respiratory and emerging Infection Consortium (ISARIC) and the Platform for European Preparedness Against (Re-)emerging Epidemics (PREPARE) that assure and prepare an agile research response to outbreak-prone infectious diseases (https:/www.prepareeurope.eu/; https://isaric.tghn.org/).

\section{Funding application and ethical permission}

The time between application and receipt of funding and between submission and acceptance by an ethical review board is usually several months. ${ }^{8} 9$ This sequential process therefore often leads to significant delays. A recent example is the Zika virus pandemic that peaked at the beginning of 2016 when WHO declared it a Public Health Emergency of International Concern. By the time the Zika virus research consortia could initiate their work with funding from the European Union in October 2016, the peak of the epidemic had passed, and most participating researchers still needed to go through ethical approval ${ }^{10}$ (figure 1).

Fortunately, there are already initiatives in place to accelerate the process of grant application and ethical review during an outbreak. The Global Research Collaboration for Infectious Disease Preparedness (GLOPID-R) joins together major public and private research funding organisations to facilitate the mobilisation of resources and the immediate start of critical research in an outbreak situation (https://www. glopid-r.org/). ${ }^{11} 12$ Legal, ethical, logistical 
Suspected and confirmed ZIKV cases in the Americas

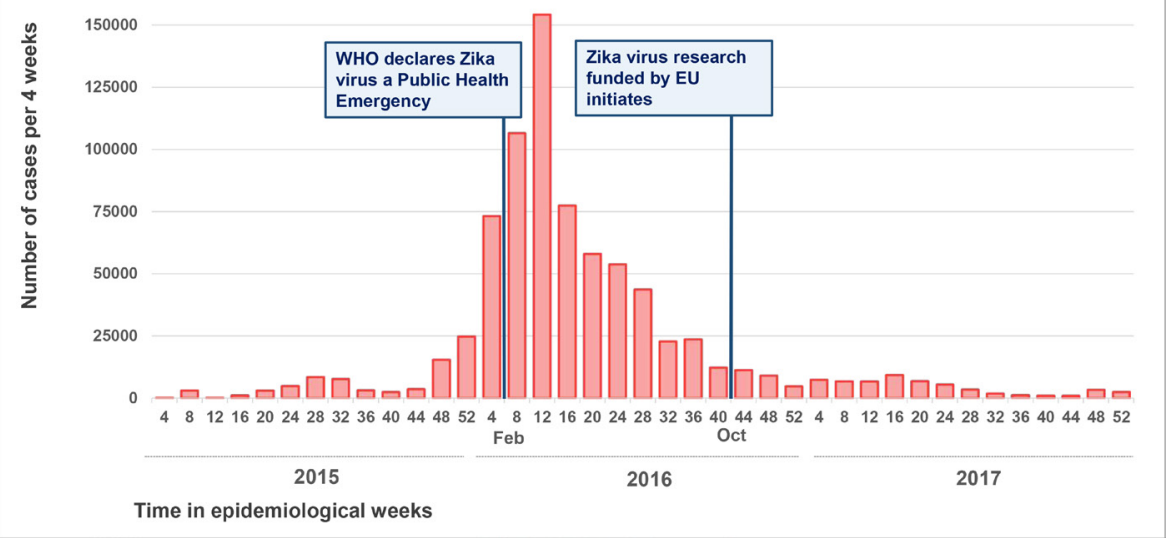

Figure 1 Suspected and confirmed ZIKV cases reported in the Americas by the WHO between January 2015 and December 2017, displayed per four epidemiological weeks. ${ }^{25}$ In October 2016, the ZikaPLAN consortium was able to initiate with funding from the EU as part of the Horizon 2020 programme. ${ }^{10} \mathrm{EU}$, European Union.

and administrative barriers that delay a research response at the peak of a health crisis could be addressed by making funding available during interpandemic periods, which can be used to develop standardised study protocols and research networks, with an additional budget to support infrastructure when the next outbreak occurs.

The idea of a 'central' or 'universal' institutional review board (IRB) in which institutional review could be fasttracked in situations of emergent infectious diseases has recently been launched by the National Institutes of Health. Furthermore, in some jurisdictions, events of public health emergency can bypass complete IRB approval, thus shortening the time to implementation. ${ }^{13} 14$

\section{Collecting and sharing data and biosamples}

As GBS is a rare disease (1-2 per 100000 per year), a multicentre or even multinational approach is generally necessary to capture a sufficient number of cases to provide evidence of an association and describe the clinical phenotype. ${ }^{15}$ Setting up a multicentre study is time-consuming, and increasingly complex privacy regulations further restrict the sharing of data and biosamples between institutions. Operational consortia allow for the continued multicentre collection of data and samples during an epidemic, although sharing of biosamples often still requires material transfer agreements. Having preapproved protocols and agreements ready for use upfront could accelerate this process.

\section{CHALLENGES AND OPPORTUNITIES IN DIAGNOSIS AND MANAGEMENT}

In case of a sudden increase in patients with GBS, clinicians with limited expertise in GBS may need to manage these patients, and availability of facilities and resources may run out. We expect limitations mainly in ICU beds and rehabilitation care, as this was also reported during the Zika virus outbreak in Brazil. ${ }^{16}$ These limitations are especially important in lowresource countries that often have suboptimal or malfunctional healthcare systems, a lack of health professionals and are hot spots for outbreaks of emerging infectious diseases. ${ }^{17}$ Here, we provide recommendations on how to safeguard good quality diagnosis and management of GBS during a pandemic.

\section{Guideline for management of GBS}

Diagnosis, treatment and monitoring of GBS can be complicated as patients may present with non-specific symptoms and vary with respect to clinical severity, treatment response and outcome. ${ }^{18}$ Furthermore, there are several diseases that can be difficult to distinguish from GBS, such as critical illness neuropathy, which is now especially important as many patients are admitted to the ICU for extended periods of time due to coronavirus disease 2019 (COVID-19). Recently a 10-step evidencebased consensus guideline for GBS was developed in response to the Zika virus outbreak. ${ }^{19}$ This guideline was designed to be compact and easy-to-use and applicable in all healthcare settings. An online version of the guideline is supported by The Global Health Network (https://rede. tghn.org/gbs-flowchart-sample/introduction-gbs/). Its use may help improve the management of GBS during an outbreak.

\section{Availability of resources}

The two proven effective therapeutics for GBS, intravenous immunoglobulin (IVIg) and plasmapheresis, are expensive, and unaffordable for many patients in lowresource countries. Furthermore, demand for IVIg has tripled in the past decades, and shortages may occur in times of crisis. ${ }^{20}$ New and affordable treatment options for GBS are therefore warranted. A pilot study on small volume plasma exchange showed potential, but the therapeutic efficacy needs to be determined. ${ }^{22}$

The COVID-19 pandemic has made it apparent that upscaling availability of ICU beds is necessary to prepare for future outbreaks of infectious diseases that cause acute respiratory distress. Prediction models for respiratory failure in patients with GBS, such as the Erasmus GBS Respiratory Insufficiency Score, may further relieve pressure from ICU facilities but need to be validated in non-Western countries. $^{23}$ Now that more patients are recovering from COVID-19, lack of caretakers and beds in rehabilitation units is also increasingly becoming a problem. ${ }^{24}$ Upscaling availability is imperative to cope with this new wave of patients and will also be of use in a future outbreak of GBS.

\section{CONCLUSION}

In the past decade, multiple pandemics of infectious diseases have been linked to increased incidence of GBS. Epidemics will continue to occur, and it is vital to advance preparedness in research and clinical management of GBS in an outbreak setting.

\section{Twitter Sonja E Leonhard @LeonhardSonja}

Contributors BCJ and SEL: study concept and design, draft of the first manuscript and interpretation of the data, revision of the manuscript for intellectual content. All other authors: revision of the manuscript for intellectual content.

Funding SEL is supported by a grant from the European Union (Horizon 2020, ZikaPLAN grant agreement no. 734584). HPE reports funding received from two grants from the European Union (Horizon 2020, ZikaPLAN, grant agreement no. 734584 and GloPID-R Sec 2, grant agreement no. 874667), and from GBS CIDP Foundation International. $\mathrm{BCJ}$ is supported by a grant from the European Union (Horizon 2020, ZikaPLAN grant agreement no. 734584) and has received funding from Prinses Beatrix Spierfonds, GBSCIDP Foundation International, CSL-Behring, Grifols, Annexon and Hansa Biopharma.

Competing interests DRC has received consultancy honoraria from Mitsubishi Tanabe Pharma Corporation, Alnylam Pharmaceuticalsc, Hansa Medical Ab,

Pledpharma, Momenta Pharmaceuticals., CSL Behring, 
Sanofi-Aventis Recherche and Developpement, Pfizer Inc, Seattle Geneticsc, Octapharma Ag, Grifols, S.A., Cigna, Pharnext Sa, Annexon Biosciences, UCB Pharma, Boehringer Ingelheim, Biotest Pharmaceuticals., Argenx, Cytomx Therapeutics, Passagebio Pharma, Seattle Genetics, Astrazeneca Pharmaceuticals, Genentech, Merrimack Pharmaceuticals, Disarm Therapeutics, Levicept, Amgen. Johns Hopkins University has the rights to The Total Neuropathy Score for which DRC receives royalty payments.

Patient consent for publication Not required.

Provenance and peer review Not commissioned; externally peer reviewed.

\section{() \\ OPEN ACCESS}

Open access This is an open access article distributed in accordance with the Creative Commons Attribution 4.0 Unported (CC BY 4.0) license, which permits others to copy, redistribute, remix, transform and build upon this work for any purpose, provided the original work is properly cited, a link to the licence is given, and indication of whether changes were made. See: https://creativecommons.org/licenses/by/ 4.0\%.

(c) Author(s) (or their employer(s)) 2020. Re-use permitted under CC BY. Published by BMJ.

\section{Check for updates}

To cite Leonhard SE, Cornblath DR, Endtz HP, et al. J Neurol Neurosurg Psychiatry 2020;91:1027-1029.

Received 15 June 2020

Accepted 20 June 2020

Published Online First 30 July 2020

J Neurol Neurosurg Psychiatry 2020;91:1027-1029. doi:10.1136/jnnp-2020-324230

\section{ORCID iD}

Sonja E Leonhard http://orcid.org/0000-0003-48326926

\section{REFERENCES}

1 Zhao H, Shen D, Zhou H, et al. Guillain-Barré syndrome associated with SARS-CoV-2 infection: causality or coincidence? Lancet Neurol 2020;19:383-4.

2 Styczynski AR, Malta JMAS, Krow-Lucal ER, et al. Increased rates of Guillain-Barré syndrome associated with Zika virus outbreak in the Salvador metropolitan area, Brazil. PLoS Negl Trop Dis 2017;11:e0005869.

3 Toscano G, Palmerini F, Ravaglia S, et al. Guillain-Barré syndrome associated with SARS-CoV-2. N Engl I Med 2020. doi:10.1056/NEJMc2009191. [Epub ahead of print: 17 Apr 2020]

4 Doets AY, Verboon C, van den Berg B, et al. Regional variation of Guillain-Barré syndrome. Brain 2018;141:2866-77

5 Muñoz LS, Barreras P, Lizarazo J, et al. Neuroviruses emerging in the Americas study (NEAS): the Colombian experience during the 2016 outbreak of Zika virus infection (N4.002). Neurology 2017:88:N4.002.

6 International GBS Outcome Study Consortium. International GBS outcome study Consortium. Available: www.gbsstudies.erasmusmc.nl

7 Jacobs BC, van den Berg B, Verboon C, et al. International Guillain-Barré syndrome outcome study: protocol of a prospective observational cohort study on clinical and biological predictors of disease course and outcome in Guillain-Barré syndrome. J Peripher Nerv Syst 2017:22:68-76.

8 Christie DRH, Gabriel GS, Dear K. Adverse effects of a multicentre system for ethics approval on the progress of a prospective multicentre trial of cancer treatment: how many patients die waiting? Intern Med J 2007;37:070807093947002

9 Schnitzbauer AA, Lamby PE, Mutzbauer I, et al. Procedures for ethical review for clinical trials within the EU. BMJ 2009;338:b1893.

10 Wilder-Smith A, Preet R, Renhorn KE, et al. ZikaPLAN: Zika preparedness Latin American network. Glob Health Action 2017:10:1398485.

11 Gobat N, Amuasi J, Yazdanpanah Y, et al. Advancing preparedness for clinical research during infectious disease epidemics. ERJ Open Res 2019;5. doi:10.1183/23120541.00227-2018. [Epub ahead of print: 20 May 2019].

12 World Health Organization. World experts and funders set priorities for COVID-19 research, 2020.

13 Schopper D, Dawson A, Upshur R, et al. Innovations in research ethics governance in humanitarian settings. BMC Med Ethics 2015;16:10.
14 Scott AM, Kolstoe S, Ploem MCC, et al. Exempting lowrisk health and medical research from ethics reviews: comparing Australia, the United Kingdom, the United States and the Netherlands. Health Res Policy Syst 2020;18:11.

15 Sejvar JJ, Baughman AL, Wise M, et al. Population incidence of Guillain-Barré syndrome: a systematic review and meta-analysis. Neuroepidemiology 2011;36:123-33.

16 Leonhard SE, Conde RM, de Assis Aquino Gondim $F$, et al. Diagnosis and treatment of Guillain-Barré syndrome during the Zika virus epidemic in Brazil: a national survey study. J Peripher Nerv Syst 2019;24:340-7

17 Jones KE, Patel NG, Levy MA, et al. Global trends in emerging infectious diseases. Nature 2008:451:990-3.

18 Verboon C, van Doorn PA, Jacobs BC. Treatment dilemmas in Guillain-Barré syndrome. J Neurol Neurosurg Psychiatry 2017;88:346-52.

19 Leonhard SE, Mandarakas MR, Gondim FAA et al. Diagnosis and management of GuillainBarré syndrome in ten steps. Nat Rev Neurol 2019;15:671-83

20 Edington HJ, Sutton KS, Bennett C, et al. Dealing with a critical national shortage-Approaches to triaging immune globulin supply in pediatric hematology and oncology. Pediatr Blood Cancer 2020;67:e28260.

21 Kerr J, Quinti I, Eibl M, et al. Is dosing of therapeutic immunoglobulins optimal? A review of a three-decade long debate in Europe. Front Immunol 2014:5:629.

22 Islam MB, Islam Z, Rahman S, et al. Small volume plasma exchange for Guillain-Barré syndrome in resource poor settings: a safety and feasibility study. Pilot Feasibility Stud 2017;3:40.

23 Walgaard C, Lingsma HF, Ruts L, et al. Prediction of respiratory insufficiency in Guillain-Barré syndrome. Ann Neurol 2010;67:781-7.

24 Boldrini P, Bernetti A, Fiore $P$, et al. Impact of COVID-19 outbreak on rehabilitation services and physical and rehabilitation medicine (PRM) physicians' activities in Italy. An official document of the Italian PRM Society (SIMFER). Eur J Phys Rehabil Med 2020

25 Pan American Health Organization. PLISA health information platform for the Americas, cumulative Zika virus disease cases. Available: https://www.paho. org/data/index.php/en/?option=com content\&view= article\&id=524:zika-weekly-en\&ltemid=352 